\title{
On the Quantum Mechanics for One Photon
}

\author{
Yapeng $\mathrm{Hu}^{1 *}, \quad$ Weigang $\mathrm{Qiu}^{2 \dagger}, \quad$ Hongbao Zhang ${ }^{1,3,4 \ddagger}$ \\ ${ }^{1}$ Department of Physics, Beijing Normal University, Beijing, 100875, China \\ ${ }^{2}$ Department of Physics, Huzhou Teachers College, Zhejiang, 313000, China \\ ${ }^{3}$ Department of Astronomy, Beijing Normal University, Beijing, 100875, China \\ ${ }^{4}$ CCAST(World Laboratory), P.O. Box 8730, Beijing, 100080, China
}

April 10, 2018

\begin{abstract}
This paper revisits the quantum mechanics for one photon from the modern viewpoint and by the geometrical method. Especially, besides the ordinary (rectangular) momentum representation, we provide an explicit derivation for the other two important representations, called the cylindrically symmetrical representation and the spherically symmetrical representation, respectively. These other two representations are relevant to some current photon experiments in quantum optics. In addition, the latter is useful for us to extract the information on the quantized black holes. The framework and approach presented here are also applicable to other particles with arbitrary mass and spin, such as the particle with spin $\frac{1}{2}$.
\end{abstract}

\section{Introduction}

From the modern viewpoint, relativistic quantum mechanics originates from the natural marriage of special relativity and quantum theory: The Hilbert space for one particle quantum wave functions forms the unitary representation of the Poincare group, which is the isometric transformation group of the Minkowski spacetime. Especially, as realized on the Minkowski spacetime, the quantum wave

*Email: yphu@mail.bnu.edu.cn

$\dagger$ Email: wgqiu@hutc.zj.cn

‡Email: hbzhang@pkuaa.edu.cn 
functions need to satisfy the field equation of motion 1, 2, 3, 4, 5. Not only does it provide a basis for relativistic quantum field, i.e., the quantum field operator is just defined on the Fock space associated with the Hilbert space of one particle states, but relativistic quantum mechanics itself is of significance in those cases which do not involve particle creation and annihilation, such as free propagations for in states before interaction and out states after interaction. It is here that relativistic quantum mechanics demonstrates its most striking properties such as quantum superposition and quantum entanglement, and thus acquires many invaluable applications such as quantum information and quantum computation [6]. Moreover, the information on the interaction can be extracted by comparing out states with in states.

Obviously, light occupies a special position in our attempts to understand nature both relativistically and quantum mechanically. It was light that initiated the great birth of both special relativity and quantum theory. Furthermore, the quantum mechanics on the photon and its interaction with matters has been developed into an individual discipline with wide applications, now called quantum optics [7]. By the geometric method, this paper is mainly intended to revisit the quantum mechanics for one photon from the modern viewpoint mentioned in the beginning. In particular, besides the momentum representation, we explicitly provide the other two important representations, i.e., the cylindrically symmetrical representation and the spherically symmetrical one. These other two representations are very relevant to some of current research in quantum optics [8, 9, 10, 11]. Especially, the latter is significant to the multi-pole radiation and electromagnetic scattering around such a central potential as the Schwarzschild black hole. Furthermore, based on the Hod's corresponding principle, it acquires a new application in the quantized black holes $[12]$.

It is worth noting that although all the three representations are known ${ }^{1}$, they are treated here in a uniform framework and on an equal footing. Especially, for the derivation of the later two representations, our method is obviously different from that used before 13, 14. We here employ the spin weighted harmonics functions and the corresponding spin weighted raising(lowering) operators, which have advantage of providing a straightforward and unified formalism applicable to particles of any spin.

The paper is organized as follows. In the next section, we construct the Hilbert space for one photon states from the solutions to the Maxwell equation. Based on the Killing field realization of the Poincare Lie algebra, Section 3 well defines the relevant conserved observables on the Hilbert space for one photon states, which is thus indicated to form the unitary representation of the Poincare group. The three representations are presented in Section 4, where the explicit derivation is given. We conclude with some implications and extensions in Section 5.

Our notation and conventions follow those in [3]. In particular, the index is raised or lowered by

${ }^{1}$ The first and third can be found in many of advanced textbooks such as [13], but the second was obtained for the first time in 14 . 
the Minkowski metric $\eta_{a b}$. We denote the covariant derivative and volume element compatible with the metric by $\nabla_{a}$ and $\epsilon_{a b c d}$ respectively. The d'Alembertian is defined as $\square=\nabla_{a} \nabla^{a}$. The Lorentz coordinate system is specially denoted by $\left\{x^{\mu} \mid \mu=0,1,2,3\right\}$, and the spatial vectors are indicated by letters in boldface.

\section{The Hilbert Space for One Photon States}

Start with the source free Maxwell equation on the Minkowski spacetime

$$
\begin{aligned}
\nabla_{[a} F_{b c]} & =0 \\
\nabla^{a} F_{a b} & =0
\end{aligned}
$$

where $F_{a b}$ is a skew tensor field, called field strength. It is obvious that the solutions to the Maxwell equation form a complex vector space, denoted by $H$. (More precisely, we define $H$ to be the complex vector space of solutions which vanish rapidly at spatial infinity.) To introduce an inner product on our complex vector space, we first define a conserved current as

$$
j_{a}\left[A, A^{\prime}\right]=i\left[\bar{F}_{a b} A^{\prime b}-\bar{A}^{b} F_{a b}^{\prime}\right]
$$

where $A_{a}$ is the vector potential, satisfying

$$
F_{a b}=2 \nabla_{[a} A_{b]}
$$

Whence the inner product can be defined as

$$
\left(F, F^{\prime}\right)=\left(A, A^{\prime}\right)=\int_{\Sigma} j^{a}\left[A, A^{\prime}\right] \epsilon_{a b c d}
$$

Note that the conservation of $j_{a}\left[A, A^{\prime}\right]$ implies that this inner product is independent of choice of the Cauchy surface $\Sigma^{2}$. Thus, for the later convenience, we choose the surface of constant $x^{0}$ as $\Sigma$ once and for all. Moreover, Eqn.(41) can be written as

$$
\left(F, F^{\prime}\right)=\left(A, A^{\prime}\right)=\int_{\Sigma}\left(\frac{\partial}{\partial x^{0}}\right)^{a} j_{a}\left[A, A^{\prime}\right] \tilde{\epsilon}_{b c d}
$$

where $\tilde{\epsilon}_{b c d}=\left(\frac{\partial}{\partial x^{0}}\right)^{a} \epsilon_{a b c d}$ is the induced spatial volume element on $\Sigma$.

\footnotetext{
${ }^{2}$ This point also implies the unitarity of the evolution of source free fields.
} 
In addition, by Eqn.(2), Eqn.(15), and the second part of Eqn.(11), the Stokes theorem shows that the inner product is invariant under gauge transformations

$$
\begin{aligned}
& A_{a} \rightarrow A_{a}+\nabla_{a} \Lambda, \\
& A_{a}^{\prime} \rightarrow A_{a}^{\prime}+\nabla_{a} \Lambda^{\prime},
\end{aligned}
$$

where $\Lambda$ and $\Lambda^{\prime}$ are both arbitrary scalar fields. However, this inner product is not always nonnegative on our whole complex vector space. We next restrict $H$ to its sub-vector space which guarantees the non-negativity of the above inner product. We denote this sub-vector space by $H^{+}$, which is just the Hilbert space for one photon states.

\section{Conserved Observables from The Poincare Lie Algebra}

As is well known, the Poincare Lie algebra can be realized by the Killing vector fields on the Minkowski spacetime as follow

$$
\begin{aligned}
P_{\mu} & =i\left(\frac{\partial}{\partial x^{\mu}}\right)^{a} \\
M_{\mu \nu} & =i\left[x_{\mu}\left(\frac{\partial}{\partial x^{\nu}}\right)^{a}-x_{\nu}\left(\frac{\partial}{\partial x^{\mu}}\right)^{a}\right] .
\end{aligned}
$$

According to the fact that the covariant derivative commutes with the Lie derivatives via Killing vector fields, the operators from the Poincare Lie algebra, i.e.

$$
\begin{aligned}
\hat{P}^{\mu} F_{a b} & =£_{P^{\mu}} F_{a b}, \\
\hat{M}_{\mu \nu} F_{a b} & =£_{M_{\mu \nu}} F_{a b},
\end{aligned}
$$

are well defined on $H$. Moreover, it can be also shown that they are well defined on the Hilbert space for one photon states indeed ${ }^{3}$. Later, employing the Leibnitz rule, the conservation of $j_{a}\left[A, A^{\prime}\right]$, and the Stokes theorem, we find that the above operators are hermitian with respect to the inner product (5). In addition, since the inner product (5) is independent of the choice of $\Sigma$, the above operators is also conserved observables. Furthermore, taking into account $\left[£_{u}, £_{v}\right]=£_{[u, v]}$ with $u$ and $v$ arbitrary vector fields, we can obtain

$$
\begin{gathered}
{\left[\hat{P}_{\mu}, \hat{P}_{\nu}\right]=0} \\
{\left[\hat{P}_{\mu}, \hat{M}_{\rho \sigma}\right]=2 i \eta_{\mu[\rho} \hat{P}_{\sigma]},}
\end{gathered}
$$

\footnotetext{
${ }^{3}$ It seems easier to prove in the rectangular momentum representation.
} 


$$
\left[\hat{M}_{\mu \nu}, \hat{M}_{\rho \sigma}\right]=2 i\left(\eta_{\mu[\rho} \hat{M}_{\sigma] \nu}-\eta_{\nu[\rho} \hat{M}_{\sigma] \mu}\right) .
$$

Here, $\hat{P}^{\mu}$ is the four-momentum operator. By Eqn(11), we have

$$
\hat{P}_{\mu} \hat{P}^{\mu}=-\square=0,
$$

which shows that the eigenvalue of the four-momentum operator is null. Furthermore, $\left\{\hat{L}_{1} \equiv\right.$ $\left.\hat{M}_{23}, \hat{L}_{2} \equiv \hat{M}_{31}, \hat{L}_{3} \equiv \hat{M}_{12}\right\}$ are the total angular momentum operators.

We next introduce the Pauli-Lubanski spin vector operator

$$
\hat{S}_{\mu}=\frac{1}{2} \epsilon_{\mu \nu \rho \sigma} \hat{P}^{\nu} \hat{M}^{\rho \sigma} .
$$

Resorting to Eqn.(1) and after a straightforward calculation, we can obtain ${ }^{4}$

$$
\hat{S}_{\mu}=\hat{P}_{\mu} \hat{S},
$$

where $\hat{S}$ is the helicity operator, defined by

$$
\hat{S} F_{a b}=(-i)^{*} F_{a b}=-\frac{i}{2} \epsilon_{a b c d} F^{c d} .
$$

Based on the fact that the Lie derivatives via Killing vector fields annihilate the volume element, $\hat{S}$ commutes with both $\hat{P}_{\mu}$ and $\hat{M}_{\mu \nu}$. Furthermore, we have

$$
\hat{S}^{2}=1
$$

which implies that the possible eigenvalue of the helicity operator takes \pm 1 .

\section{Three Representations in the Coulomb Gauge}

In this section, we shall employ the vector potential in the Coulomb gauge. In terms of the vector potential, the Maxwell equation can be written as

$$
\square A_{a}=0,
$$

where the Coulomb gauge

$$
\begin{aligned}
\nabla^{a} A_{a} & =0, \\
\left(\frac{\partial}{\partial x^{0}}\right)^{a} A_{a} & =0,
\end{aligned}
$$

\footnotetext{
${ }^{4}$ The reader is suggested to follow the steps described in [15].
} 
has been employed. In this case, the inner product (5) is equivalent to

$$
\left(F, F^{\prime}\right)=\left(A, A^{\prime}\right)=\int_{\Sigma}\left(\frac{\partial}{\partial x^{0}}\right)^{a} j_{a}^{\prime}\left[A, A^{\prime}\right] \tilde{\epsilon}_{b c d},
$$

where the conserved current

$$
\left.j_{a}^{\prime}\left[A, A^{\prime}\right]=i\left[\nabla_{a} \bar{A}_{b}\right) A^{\prime b}-\bar{A}^{b} \nabla_{a} A_{b}^{\prime}\right] .
$$

Later, according to the commutation relations in the last section, we can choose $\left\{\hat{P}^{1}, \hat{P}^{2}, \hat{P}^{3}, \hat{S}\right\}$ as a complete observable set, which forms the ordinary rectangular momentum representation. Similarly, the complete observable set $\left\{\hat{P}^{0}, \hat{P}^{3}, \hat{L}_{3}, \hat{S}\right\}$ forms the cylindrically symmetrical representation, and $\left\{\hat{P}^{0}, \hat{\mathbf{L}}^{2}, \hat{L}_{3}, \hat{S}\right\}$ forms the spherically symmetrical representation.

\subsection{The Rectangular Momentum Representation}

Since the details of the rectangular momentum representation have appeared in the literature, we will only recall the main results without entering into the explicit derivations. Firstly, according to Eqn.(17) and Eqn.(18), any vector potential can be written as

$$
\begin{aligned}
A_{a}(x)= & \frac{1}{\sqrt{(2 \pi)^{3}}}\left\{\int_{p^{0}>0} \frac{d^{3} \mathbf{p}}{p^{0}}\left[\dot{A}_{+}(\mathbf{p})\left(\varepsilon^{+}\right)_{a}(\mathbf{p})+\dot{A}_{-}(\mathbf{p})\left(\varepsilon^{-}\right)_{a}(\mathbf{p})\right] e^{-i p_{b} x^{b}}\right. \\
& \left.+\int_{p^{0}<0} \frac{d^{3} \mathbf{p}}{p^{0}}\left[\grave{A}_{+}(\mathbf{p})\left(\varepsilon^{+}\right)_{a}(\mathbf{p})+\grave{A}_{-}(\mathbf{p})\left(\varepsilon^{-}\right)_{a}(\mathbf{p})\right] e^{-i p_{b} x^{b}}\right\} .
\end{aligned}
$$

Here $p^{a}=p^{u}\left(\frac{\partial}{\partial x^{\mu}}\right)^{a}$ is a constant real null vector field, and $x^{a}=x^{\mu}\left(\frac{\partial}{\partial x^{\mu}}\right)^{a}$ is the position vector field. In addition, $\left(\varepsilon^{ \pm}\right)_{a}(\mathbf{p})$ are constant null fields and complex conjugate with each other, satisfying

$$
\begin{aligned}
p^{a}\left(\varepsilon^{ \pm}\right)_{a}(\mathbf{p}) & =0 \\
\left(\frac{\partial}{\partial x^{0}}\right)^{a}\left(\varepsilon^{ \pm}\right)_{a}(\mathbf{p}) & =0, \\
\epsilon_{a b c d} & =-\frac{i}{p^{0}}\left(d x^{0}\right)_{a} \wedge p_{b} \wedge\left(\varepsilon^{+}\right)_{c}(\mathbf{p}) \wedge\left(\varepsilon^{-}\right)_{d}(\mathbf{p}) .
\end{aligned}
$$

Later, substituting Eqn.(21) into Eqn.(19), we have

$$
\begin{aligned}
\left(F, F^{\prime}\right)= & \left(A, A^{\prime}\right)=\int d^{3} \mathbf{x}\left(\frac{\partial}{\partial x^{0}}\right)^{a} j_{a}^{\prime}\left[A, A^{\prime}\right] \\
= & 2\left\{\int_{p^{0}>0} \frac{d^{3} \mathbf{p}}{p^{0}}\left[\bar{A}_{+}(\mathbf{p}) \hat{A}^{\prime}{ }_{+}(\mathbf{p})+\bar{A}_{-}(\mathbf{p}) \dot{A}^{\prime}{ }_{-}(\mathbf{p})\right]\right. \\
& \left.+\int_{p^{0}<0} \frac{d^{3} \mathbf{p}}{p^{0}}\left[\bar{A}_{+}(\mathbf{p}) \grave{A}_{+}^{\prime}(\mathbf{p})+\bar{A}_{-}(\mathbf{p}) \grave{A}^{\prime}{ }_{-}(\mathbf{p})\right]\right\} .
\end{aligned}
$$


Whence $H^{+}$is just the positive energy solutions to the Maxwell equation, as is also what we expect. Therefore, we shall restrict us to the case of $p^{0}>0$ in all of the following discussions. Furthermore, the orthonormal basis for $H^{+}$in the rectangular momentum representation is given by

$$
|\mathbf{p}, s= \pm 1\rangle=\frac{1}{\sqrt{(2 \pi)^{3}}} \frac{1}{\sqrt{2 p^{0}}}\left(\varepsilon^{ \pm}\right)_{a}(\mathbf{p}) e^{-i p_{b} x^{b}}
$$

where $\mathbf{p}$ is the eigenvalue of three-momentum operator, and $s$ is the eigenvalue of the helicity operator.

\subsection{The Cylindrically Symmetrical Representation}

It is convenient to provide the cylindrically symmetrical representation in the cylindrical coordinate system, i.e.

$$
\begin{aligned}
x^{0} & =t, \\
x^{1} & =\varrho \cos \phi, \\
x^{2} & =\varrho \sin \phi, \\
x^{3} & =z .
\end{aligned}
$$

Whence the Minkowski metric reads

$$
d s^{2}=d t^{2}-d z^{2}-d \varrho^{2}-\varrho^{2} d \phi^{2},
$$

and

$$
\begin{aligned}
P^{0} & =i\left(\frac{\partial}{\partial t}\right)^{a}, \\
P^{3} & =-i\left(\frac{\partial}{\partial z}\right)^{a}, \\
L_{3} & =-i\left(\frac{\partial}{\partial \phi}\right)^{a} .
\end{aligned}
$$

Define a pair of null covariant vector fields as

$$
\left(\varepsilon^{\mp}\right)_{a}=\frac{1}{\sqrt{2}}\left[(d \varrho)_{a} \pm i \varrho(d \phi)_{a}\right],
$$

then according to the second part in Eqn.(18), any vector potential can be written as

$$
A_{a}=A_{z}(d z)_{a}+A_{-}\left(\varepsilon^{-}\right)_{a}+A_{+}\left(\varepsilon^{+}\right)_{a}
$$


where, $A_{z}$ has spin weight $0, A_{-}$with spin weight -1 , and $A_{+}$with spin weight 1 [16]. From

$$
\begin{aligned}
\nabla_{a}(d z)_{b} & =0, \\
\nabla_{a}\left(\varepsilon^{-}\right)_{b} & =\frac{1}{\sqrt{2} \varrho}\left[\left(\varepsilon^{+}\right)_{a}-\left(\varepsilon^{-}\right)_{a}\right]\left(\varepsilon^{-}\right)_{b}, \\
\nabla_{a}\left(\varepsilon^{+}\right)_{b} & =\frac{1}{\sqrt{2} \varrho}\left[\left(\varepsilon^{-}\right)_{a}-\left(\varepsilon^{+}\right)_{a}\right]\left(\varepsilon^{+}\right)_{b},
\end{aligned}
$$

it can be shown that the Maxwell equation reads

$$
\begin{aligned}
\square A_{a}= & \left(-\bar{\varnothing} ð A_{z}+\frac{\partial^{2} A_{z}}{\partial t^{2}}-\frac{\partial^{2} A_{z}}{\partial z^{2}}\right)(d z)_{a} \\
& +\left(-\bar{\varnothing} ð A_{-}+\frac{\partial^{2} A_{-}}{\partial t^{2}}-\frac{\partial^{2} A_{-}}{\partial z^{2}}\right)\left(\varepsilon^{-}\right)_{a} \\
& +\left(-\bar{\varnothing} ð A_{+}+\frac{\partial^{2} A_{+}}{\partial t^{2}}-\frac{\partial^{2} A_{+}}{\partial z^{2}}\right)\left(\varepsilon^{+}\right)_{a}=0,
\end{aligned}
$$

together with

$$
\nabla^{a} A_{a}=-\frac{\partial A_{z}}{\partial z}+\frac{1}{\sqrt{2}}\left(ð A_{-}+\bar{\partial} A_{+}\right)=0 .
$$

Here $\partial, \bar{\partial}$ are operators acting on a quantity $f$ with spin weight $n$, i.e.

$$
\begin{aligned}
& \partial f=-\left(\frac{\partial}{\partial \varrho}+\frac{i}{\varrho} \frac{\partial}{\partial \phi}-\frac{n}{\varrho}\right) f, \\
& \bar{\partial} f=-\left(\frac{\partial}{\partial \varrho}-\frac{i}{\varrho} \frac{\partial}{\partial \phi}+\frac{n}{\varrho}\right) f .
\end{aligned}
$$

Then, $\supset f$ and $\bar{\partial} f$ have spin weight $n+1$ and $n-1$, respectively [16]. Later, it is easy to check that the Lie derivatives of $\left\{(d z)_{a},\left(\varepsilon^{\mp}\right)_{a}\right\}$ via $\left\{P^{0}, P^{3}, L_{3}\right\}$ all vanish, thus the simultaneous eigensolutions of $\left\{\hat{P}^{0}, \hat{P}^{3}, \hat{L}_{3}\right\}$ to Eqn.(131) with the corresponding eigenvalue $\left\{p^{0}, p^{3}, m\right\}$ must take the form

$$
\begin{aligned}
& A_{z}=a_{00} Z_{\alpha m}(\varrho, \phi) e^{-i\left(p_{0} t+p_{3} z\right)}, \\
& A_{-}=a_{--1} Z_{\alpha m}(\varrho, \phi) e^{-i\left(p_{0} t+p_{3} z\right)} \\
& A_{+}=a_{+1} Z_{\alpha m}(\varrho, \phi) e^{-i\left(p_{0} t+p_{3} z\right)}
\end{aligned}
$$

where $a_{0}, a_{-}$, and $a_{+}$are all constant coefficients; ${ }_{n} Z_{\alpha m}$ is the spin-weighted cylindrical harmonics with spin weight $\mathrm{n}$ such that [16]

$$
\begin{array}{r}
\partial_{n} Z_{\alpha m}=\alpha_{n+1} Z_{\alpha m}, \\
\overline{\mathrm{\partial}}_{n} Z_{\alpha m}=-\alpha_{n-1} Z_{\alpha m}, \\
\hat{L}_{3 n} Z_{\alpha m}=m_{n} Z_{\alpha m}
\end{array}
$$


with $\alpha=\sqrt{p_{0}^{2}-p_{3}^{2}}$. Moreover, by the boundary condition, here $p_{3}$ is a real constant, and

$$
{ }_{n} Z_{\alpha m}=J_{m+n}(\alpha \varrho) e^{i m \phi},
$$

where $J_{m+n}$ is the first kind of Bessel function of order $m+n$ with $\alpha \geq 0$ and $m$ an integer.

Substituting Eqn.(34) into Eqn.(32), we have

$$
i p_{3} a_{0}+\frac{\alpha}{\sqrt{2}}\left(a_{-}-a_{+}\right)=0
$$

Next combine it with the eigenequations of the helicity operator, i.e.

$$
\begin{aligned}
& i p_{0} a_{0}-s \frac{\alpha}{\sqrt{2}}\left(a_{-}+a_{+}\right)=0, \\
& i\left(p_{0}-s p_{3}\right) a_{+}+s \frac{\alpha}{\sqrt{2}} a_{0}=0, \\
& i\left(p_{0}+s p_{3}\right) a_{-}+s \frac{\alpha}{\sqrt{2}} a_{0}=0,
\end{aligned}
$$

where $s= \pm 1$ is the eigenvalue of the helicity operator. Thus we have

$$
\begin{aligned}
& a_{-}=\frac{i s a_{0}}{\sqrt{2} \alpha}\left(p_{0}-s p_{3}\right) \\
& a_{+}=\frac{i s a_{0}}{\sqrt{2} \alpha}\left(p_{0}+s p_{3}\right) .
\end{aligned}
$$

Furthermore, note

$$
\int_{0}^{\infty} d \varrho \varrho J_{m}(\alpha \varrho) J_{m}\left(\alpha^{\prime} \varrho\right)=\frac{1}{\alpha} \delta\left(\alpha-\alpha^{\prime}\right)
$$

with $\alpha \leq \alpha^{\prime}[20]$. Then it follows that the orthonormal basis with respect to the inner product (19) in the cylindrically symmetrical representation reads

$$
\begin{aligned}
\left|p^{0}, p^{3}, m, s\right\rangle= & \frac{\alpha}{4 \pi p_{0}}\left\{J_{m}(\alpha \varrho) e^{i m \phi} e^{-i\left(p_{0} t+p_{3} z\right)}(d z)_{a}\right. \\
& +\frac{i}{\sqrt{2} \alpha}\left[\left(s p_{0}-p_{3}\right) J_{m-1}(\alpha \varrho) e^{i m \phi} e^{-i\left(p_{0} t+p_{3} z\right)}\left(\varepsilon^{-}\right)_{a}\right. \\
& \left.\left.+\left(s p_{0}+p_{3}\right) J_{m+1}(\alpha \varrho) e^{i m \phi} e^{-i\left(p_{0} t+p_{3} z\right)}\left(\varepsilon^{+}\right)_{a}\right]\right\}
\end{aligned}
$$

which satisfies

$$
\left\langle p^{0}, p^{3}, m, s \mid p^{\prime 0}, p^{\prime 3}, m^{\prime}, s^{\prime}\right\rangle=\delta\left(p^{0}-p^{\prime 0}\right) \delta\left(p^{3}-p^{\prime 3}\right) \delta_{m m^{\prime}} \delta_{s s^{\prime}}
$$

Finally, we would like to point out that $\left|p^{0}, p^{3}, m, s\right\rangle$ vanishes for $m \neq \pm 1$ in the case of $\alpha=0$. 


\subsection{The Spherically Symmetrical Representation}

To provide the spherically symmetrical representation, we would like to use the spherical coordinate system, i.e.

$$
\begin{aligned}
x^{0} & =t, \\
x^{1} & =r \sin \theta \cos \varphi, \\
x^{2} & =r \sin \theta \sin \varphi, \\
x^{3} & =r \cos \theta .
\end{aligned}
$$

In this case, the Minkowski metric takes the form

$$
d s^{2}=d t^{2}-d r^{2}-r^{2}\left(d \theta^{2}+\sin ^{2} \theta d \varphi^{2}\right)
$$

and

$$
\begin{aligned}
P^{0} & =i\left(\frac{\partial}{\partial t}\right)^{a} \\
L_{ \pm} \equiv L_{1} \pm i L_{2} & = \pm e^{ \pm i \varphi}\left[\left(\frac{\partial}{\partial \theta}\right)^{a} \pm i \cot \theta\left(\frac{\partial}{\partial \varphi}\right)^{a}\right] \\
L_{3} & =-i\left(\frac{\partial}{\partial \varphi}\right)^{a}
\end{aligned}
$$

Define a pair of null covariant vector fields as

$$
\left(\varepsilon^{\mp}\right)_{a}=\frac{r}{\sqrt{2}}\left[(d \theta)_{a} \pm i \sin \theta(d \varphi)_{a}\right]
$$

then from the second part in Eqn.(18), any vector potential reads

$$
A_{a}=A_{r}(d r)_{a}+A_{-}\left(\varepsilon^{-}\right)_{a}+A_{+}\left(\varepsilon^{+}\right)_{a}
$$

where $A_{r}$ has spin weight $0, A_{-}$with -1 , and $A_{+}$with 1[17, 18, 19]. Using

$$
\begin{aligned}
\nabla_{a}(d r)_{b} & =\frac{1}{r}\left[\left(\varepsilon^{-}\right)_{a}\left(\varepsilon^{+}\right)_{b}+\left(\varepsilon^{+}\right)_{a}\left(\varepsilon^{-}\right)_{b}\right] \\
\nabla_{a}\left(\varepsilon^{-}\right)_{b} & =\frac{1}{r}\left\{\frac{\cot \theta}{\sqrt{2}}\left[\left(\varepsilon^{+}\right)_{a}-\left(\varepsilon^{-}\right)_{a}\right]\left(\varepsilon^{-}\right)_{b}-\left(\varepsilon^{-}\right)_{a}(d r)_{b}\right\} \\
\nabla_{a}\left(\varepsilon^{+}\right)_{b} & =\frac{1}{r}\left\{\frac{\cot \theta}{\sqrt{2}}\left[\left(\varepsilon^{-}\right)_{a}-\left(\varepsilon^{+}\right)_{a}\right]\left(\varepsilon^{+}\right)_{b}-\left(\varepsilon^{+}\right)_{a}(d r)_{b}\right\}
\end{aligned}
$$


it follows that

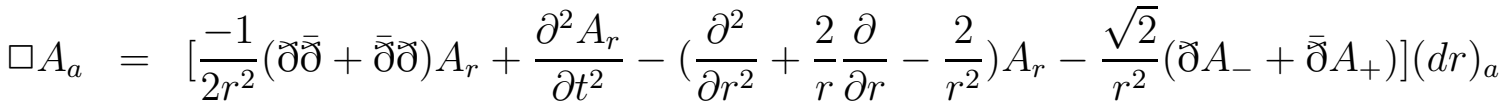

$$
\begin{aligned}
& +\left[\frac{-1}{2 r^{2}}(ð \bar{\partial}+\bar{\partial} \precsim) A_{-}+\frac{\partial^{2} A_{-}}{\partial t^{2}}-\left(\frac{\partial^{2}}{\partial r^{2}}+\frac{2}{r} \frac{\partial}{\partial r}-\frac{1}{r^{2}}\right) A_{-}+\frac{\sqrt{2}}{r^{2}} \overline{\mathrm{\partial}} A_{r}\right]\left(\varepsilon^{-}\right)_{a} \\
& +\left[\frac{-1}{2 r^{2}}(\check{\bar{\partial}}+\overline{\bar{\partial}} \check{\partial}) A_{+}+\frac{\partial^{2} A_{+}}{\partial t^{2}}-\left(\frac{\partial^{2}}{\partial r^{2}}+\frac{2}{r} \frac{\partial}{\partial r}-\frac{1}{r^{2}}\right) A_{+}+\frac{\sqrt{2}}{r^{2}} \check{\partial} A_{r}\right]\left(\varepsilon^{+}\right)_{a}=0,
\end{aligned}
$$

and

$$
\nabla_{a} A^{a}=-\left(\frac{\partial}{\partial r}+\frac{2}{r}\right) A_{r}+\frac{1}{\sqrt{2} r}\left(ð A_{-}+\overline{\mathrm{\partial}} A_{+}\right)=0 .
$$

Here $\check{\partial}, \bar{\partial}$ are operators acting on a quantity $f$ with spin weight $n$, i.e.

$$
\begin{aligned}
& \text { ळf}=-\left(\frac{\partial}{\partial \theta}+i \csc \theta \frac{\partial}{\partial \varphi}-n \cot \theta\right) f, \\
& \bar{\partial} f=-\left(\frac{\partial}{\partial \theta}-i \csc \theta \frac{\partial}{\partial \varphi}+n \cot \theta\right) f .
\end{aligned}
$$

Then, $\precsim f$ and $\bar{\partial} f$ have spin weight $n+1$ and $n-1$, respectively[19].

On the other hand, we have

$$
\begin{aligned}
& \hat{L}_{ \pm}(d r)_{a}=L_{ \pm}^{b} \partial_{b}(d r)_{a}+(d r)_{b} \partial_{a} L_{ \pm}^{b}=0 \\
& \hat{L}_{ \pm}\left(\varepsilon^{-}\right)_{a}=L_{ \pm}^{b} \partial_{b}\left(\varepsilon^{-}\right)_{a}+\left(\varepsilon^{-}\right)_{b} \partial_{a} L_{ \pm}^{b}=e^{ \pm i \varphi}\left(\varepsilon^{-}\right)_{a} \\
& \hat{L}_{ \pm}\left(\varepsilon^{+}\right)_{a}=L_{ \pm}^{b} \partial_{b}\left(\varepsilon^{+}\right)_{a}+\left(\varepsilon^{+}\right)_{b} \partial_{a} L_{ \pm}^{b}=-e^{ \pm i \varphi}\left(e^{+}\right)_{a}
\end{aligned}
$$

where $\partial_{a}$ is the ordinary derivative associated with the spherical coordinate system. Thus

$$
\begin{aligned}
\hat{L}_{ \pm} A_{a}= & \left(\hat{L}_{ \pm} A_{r}\right)(d r)_{a} \\
& +\left(\hat{L}_{ \pm} A_{-}+e^{ \pm i \varphi} \csc \theta A_{-}\right)\left(\varepsilon^{-}\right)_{a} \\
& +\left(\hat{L}_{ \pm} A_{+}-e^{ \pm i \varphi} \csc \theta A_{+}\right)\left(\varepsilon^{+}\right)_{a} .
\end{aligned}
$$

Similarly, it is easy to check that the Lie derivatives of $\left\{(d r)_{a},\left(\varepsilon^{\mp}\right)_{a}\right\}$ via $\left\{P^{0}, L_{3}\right\}$ all vanish. Whence the simultaneous eigensolutions of $\left\{\hat{P}^{0}, \hat{\mathbf{L}}^{2}, \hat{L}_{3}\right\}$ to Eqn.(49) with the corresponding eigenvalue $\left\{p^{0}, l(l+1), m\right\}$ must satisfy

$$
\begin{aligned}
& A_{r}=R_{0}(r)_{0} Y_{l m}(\theta, \varphi) e^{-i p_{0} t} \\
& A_{-}=R_{-}(r)_{-1} Y_{l m}(\theta, \varphi) e^{-i p_{0} t} \\
& A_{+}=R_{+}(r)_{1} Y_{l m}(\theta, \varphi) e^{-i p_{0} t}
\end{aligned}
$$


Here ${ }_{n} Y_{l m}$ is the spin weighted spherical harmonics with $l$ non-negative integers and $m=-l,-l+$ $1, \ldots, l$, such that

$$
\begin{aligned}
{ }_{0} Y_{l m} & =Y_{l m}, \\
\oint_{n} Y_{l m} & =\sqrt{(l-n)(l+n+1)} \\
\bar{\jmath}_{n+1} Y_{l m} & =-\sqrt{(l+n)(l-n+1)} Y_{l m}, \\
\left(\hat{L}_{ \pm}-n e^{ \pm i \varphi} \csc \theta\right)_{n} Y_{l m} & =\sqrt{(l \mp m)(l \pm m+1)_{n}} Y_{l m \pm 1}, \\
\hat{L}_{3 n} Y_{l m} & =m_{n} Y_{l m},
\end{aligned}
$$

where $Y_{l m}$ is the ordinary spherical harmonics, and ${ }_{n} Y_{l m}$ with $l<|n|$ vanishes [19].

We next substitute Eqn.(154) into Eqn.(49) to obtain the radial equations

$$
\begin{aligned}
\left(\frac{d^{2}}{d r^{2}}+\frac{2}{r} \frac{d}{d r}-\frac{2}{r^{2}}\right) R_{0}+p_{0}^{2} R_{0}-\frac{l(l+1)}{r^{2}} R_{0}+\frac{\sqrt{2 l(l+1)}}{r^{2}}\left(R_{-}-R_{+}\right) & =0, \\
\left(\frac{d^{2}}{d r^{2}}+\frac{2}{r} \frac{d}{d r}\right) R_{-}+p_{0}^{2} R_{-}-\frac{l(l+1)}{r^{2}} R_{-}+\frac{\sqrt{2 l(l+1)}}{r^{2}} R_{0} & =0, \\
\left(\frac{d^{2}}{d r^{2}}+\frac{2}{r} \frac{d}{d r}\right) R_{+}+p_{0}^{2} R_{+}-\frac{l(l+1)}{r^{2}} R_{+}-\frac{\sqrt{2 l(l+1)}}{r^{2}} R_{0} & =0 .
\end{aligned}
$$

Furthermore, Eqn.(50) requires

$$
-\left(\frac{d}{d r}+\frac{2}{r}\right) R_{0}+\frac{\sqrt{l(l+1)}}{\sqrt{2} r}\left(R_{-}-R_{+}\right)=0 .
$$

It can be shown that Eqn.(56) and Eqn.(157) are equivalent to

$$
\begin{aligned}
\left(\frac{d^{2}}{d r^{2}}+\frac{2}{r} \frac{d}{d r}\right)\left(R_{-}+R_{+}\right)+p_{0}^{2}\left(R_{-}+R_{+}\right)-\frac{l(l+1)}{r^{2}}\left(R_{-}+R_{+}\right) & =0 \\
\left(\frac{d^{2}}{d r^{2}}+\frac{4}{r} \frac{d}{d r}+\frac{2}{r^{2}}\right) R_{0}+p_{0}^{2} R_{0}-\frac{l(l+1)}{r^{2}} R_{0} & =0 \\
R_{-}-R_{+}-\frac{\sqrt{2} r}{\sqrt{l(l+1)}}\left(\frac{d}{d r}+\frac{2}{r}\right) R_{0} & =0
\end{aligned}
$$


By the boundary condition, the solutions to Eqn.(158) are given by

$$
\begin{aligned}
R_{-}+R_{+} & =b \frac{J_{l+\frac{1}{2}}\left(p_{0} r\right)}{\sqrt{p_{0} r}}, \\
R_{0} & =b_{0} \frac{J_{l+\frac{1}{2}}\left(p_{0} r\right)}{\left(\sqrt{p_{0} r}\right)^{3}}, \\
R_{-}-R_{+} & =b_{0} \frac{\sqrt{2}}{\sqrt{l(l+1)}}\left[\frac{J_{l-\frac{1}{2}}\left(p_{0} r\right)}{\sqrt{p_{0} r}}-l \frac{J_{l+\frac{1}{2}}\left(p_{0} r\right)}{\left(\sqrt{p_{0} r}\right)^{3}}\right],
\end{aligned}
$$

where $b$ and $b_{0}$ are both constant coefficients; $J_{l \pm \frac{1}{2}}$ is the first kind of Bessel function of order $l \pm \frac{1}{2}[20]$.

Substituting Eqn.(59) into the eigenequations of the helicity operator with the eigenvalue $s= \pm 1$, i.e.

$$
\begin{aligned}
i p_{0} R_{0}-s \frac{\sqrt{l(l+1)}}{\sqrt{2} r}\left(R_{-}+R_{+}\right) & =0, \\
i p_{0} R_{-}+s \frac{\sqrt{l(l+1)}}{\sqrt{2} r} R_{0}-s\left(\frac{d}{d r}+\frac{1}{r}\right) R_{-} & =0, \\
i p_{0} R_{+}+s \frac{\sqrt{l(l+1)}}{\sqrt{2} r} R_{0}+s\left(\frac{d}{d r}+\frac{1}{r}\right) R_{+} & =0,
\end{aligned}
$$

we obtain

$$
b=i s b_{0} \frac{\sqrt{2}}{\sqrt{l(l+1)}}
$$

Note

$$
\int_{4 \pi} d \varphi d \theta \sin \theta_{n} \bar{Y}_{l m n} Y_{l^{\prime} m^{\prime}}=\delta_{l l^{\prime}} \delta_{m m^{\prime}}
$$


with $l \geq|n|[19]$, and

$$
\begin{aligned}
\int_{0}^{\infty} d r r J_{l+\frac{1}{2}}\left(p_{0} r\right) J_{l+\frac{1}{2}}\left(p_{0}^{\prime} r\right) & =\frac{1}{p_{0}} \delta\left(p_{0}-p_{0}^{\prime}\right), \\
\int_{0}^{\infty} d r \frac{1}{r} J_{l+\frac{1}{2}}\left(p_{0} r\right) J_{l+\frac{1}{2}}\left(p_{0}^{\prime} r\right) & =\frac{1}{2 l+1}\left(\frac{p_{0}}{p_{0}^{\prime}}\right)^{l+\frac{1}{2}}, \\
\int_{0}^{\infty} d r J_{l-\frac{1}{2}}\left(p_{0} r\right) J_{l+\frac{1}{2}}\left(p_{0}^{\prime} r\right) & =\frac{1}{p_{0}}\left(\frac{p_{0}}{p_{0}^{\prime}}\right)^{l+\frac{1}{2}} \\
\int_{0}^{\infty} d r J_{l-\frac{1}{2}}\left(p_{0}^{\prime} r\right) J_{l+\frac{1}{2}}\left(p_{0} r\right) & =0 \\
\int_{0}^{\infty} d r J_{l-\frac{1}{2}}\left(p_{0} r\right) J_{l+\frac{1}{2}}\left(p_{0} r\right) & =\frac{1}{2 p_{0}}
\end{aligned}
$$

with $p_{0} \leq p_{0}^{\prime}$ 20. Thus it follows that the orthonormal basis with respect to the inner product (19) in the spherical symmetrical representation reads

$$
\begin{aligned}
\left|p^{0}, l, m, s\right\rangle= & \frac{\sqrt{l(l+1)}}{2 \sqrt{r}}\left\{\frac{J_{l+\frac{1}{2}}\left(p_{0} r\right)}{p_{0} r}{ }_{0} Y_{l m}(\theta, \varphi) e^{-i p_{0} t}(d r)_{a}\right. \\
& +\frac{1}{\sqrt{2 l(l+1)}}\left[\left(\frac{i s p_{0} r-l}{p_{0} r} J_{l+\frac{1}{2}}\left(p_{0} r\right)+J_{l-\frac{1}{2}}\left(p_{0} r\right)\right)_{-1} Y_{l m}(\theta, \varphi) e^{-i p_{0} t}\left(\varepsilon^{-}\right)_{a}\right. \\
& \left.\left.+\left(\frac{i s p_{0} r+l}{p_{0} r} J_{l+\frac{1}{2}}\left(p_{0} r\right)-J_{l-\frac{1}{2}}\left(p_{0} r\right)\right)_{1} Y_{l m}(\theta, \varphi) e^{-i p_{0} t}\left(\varepsilon^{+}\right)_{a}\right]\right\},
\end{aligned}
$$

which satisfies

$$
\left\langle p^{0}, l, m, s \mid p^{\prime 0}, l^{\prime}, m^{\prime}, s^{\prime}\right\rangle=\delta\left(p^{0}-p^{\prime 0}\right) \delta_{l l^{\prime}} \delta_{m m^{\prime}} \delta_{s s^{\prime}} .
$$

It is obvious that $\left|p^{0}, l, m, s\right\rangle$ vanishes in the case of $l=0$, which implies that the angular quantum number $l$ of one photon only takes positive integers.

\section{Discussions}

We would like to stress that the framework and method presented here are also applicable to other particles with arbitrary mass and spin such as neutrino and electron. In addition, after a simple modification, our results obtained here are easy to be generalized to those cavities with the suitable boundaries, which is important not only to the investigation of the Casimir effect, but also to understanding the relationship between the holographic entropy bound and local quantum field theory [21]. 


\section{Acknowledgements}

We are paticularly grateful to Prof. R. P. Geroch for his interesting exchanges of ideas and unpublished lecture notes, which directly stimulates our investigation of this project. In addition, we also thank Prof. G. F. Torres del Castillo for private communications on spin weighted harmonics functions. Y. Hu and $\mathrm{H}$. Zhang would like to acknowledge the colleagues from Gravitational Group at BNU for their endless encouragements, especially Prof. S. Pei and Dr. B. Zhou for their helpful discussions. H. Zhang owes much gratitude to Prof. H. Guo for his instructive criticisms and suggestions. Y. Hu and H. Zhang's work was supported in part by NSFC(Grant 10205002, 10373003, and 10533010). W. Qiu's work was supported by NSFC(Grant 10547116), the Science Research Fund of Huzhou Teachers College(No.KX21001) and the Science Research Fund of Huzhou City(No.KY21022).

\section{References}

[1] E. P. Wigner, Ann. Math. 40: 149(1939)

[2] V. Bargmann and E. P. Wigner, Proc. Nat. Acad. Sci. 34: 211(1948)

[3] R. P. Geroch, Special Topics in Particle Physics(Unpublished Lecture Notes, University of Texas at Austin, 1971)

[4] S. Weinberg, The Quantum Theory of Fields: Volume I(Cambridge University Press, Cambridge, 1995)

[5] S. Weinberg, hep-th/9702027

[6] A. Peres and D. R. Terno, Rev. Mod. Phys. 76: 93(2004)

[7] D. F. Walls and G. J. Milburn, Quantum Optics(Springer-Verlag, Berlin, 1994)

[8] H. He et al., Phys. Rev. Lett. 75: 826(1995)

[9] S. J. van Enk and H. J. Kimble, Phys. Rev. A 63: 023809(2001)

[10] A. Mair et al., Nature 412: 313(2001)

[11] J. Leach et al., Phys. Rev. Lett. 88: 257901(2002)

[12] S. Hod, Phys. Rev. Lett. 81: 4293(1998) 
[13] V. B. Berestetskii et al., Quantum Electrodynamics(Pergamon Press, Oxford, 1982)

[14] S. J. van Enk and G. Nienhuis, J. Mod. Optics. 41: 963(1994)

[15] A. Ashtekar, J. Math. Phys. 27: 824(1985)

[16] G. F. Torres del Castillo, J. Math. Phys. 34: 3856(1993)

[17] E. T. Newman and R. Penrose, J. Math. Phys. 7: 863(1966)

[18] J. N. Goldberg et al., J. Math. Phys. 8: 2155(1967)

[19] W. B. Campbell, J. Math. Phys. 12: 1763(1971)

[20] Z. Wang and D. Guo, Introduction to Special Functions (Peking University Press, Beijing, 2000)

[21] U. Yurtsever, Phys. Rev. Lett. 91: 041302(2003) 